\title{
Invalidly cued targets are well localized when detected
}

\section{Daniel A. Gajewski ${ }^{1} \cdot$ Junjun Zhang ${ }^{1} \cdot$ Sarah Shomstein ${ }^{1} \cdot$ Joseph C. Nah ${ }^{1} \cdot J^{\prime}$ ohn W. Philbeck ${ }^{1}$}

Published online: 18 June 2019

(C) The Psychonomic Society, Inc. 2019

\begin{abstract}
Considerable attention has been devoted to understanding how objects are localized when there is ample time and attention to detect them. However, in the real world, we often must react to, or act upon, objects that we have glimpsed only briefly and are not directly at the focus of our attention. This paper describes two experiments examining the role of attentional constraints on 2$\mathrm{D}$ (directional) localization, particularly in cases in which targets have been detected but are not within the spatial focus of attention. Targets were asterisks presented briefly $(34-150 \mathrm{~ms})$ above or below a central fixation point. Just prior to the target's appearance, a cue directed attention toward, or away from, the target. Participants indicated whether or not they saw the target, and then used a mouse to indicate the target's location. The impact of guessing was mitigated by removing trials that participants had flagged as not detected. Longer glimpses generally benefitted localization; by contrast, cue validity had very little effect on response sensitivity, bias or precision. At very brief durations, invalid cueing did result in a small increase in foveal bias. These results indicate that the directional location of objects can be extracted reasonably well from brief glimpses even with reduced attention. This directional information provides an important basis for 3-D localization of objects on the ground, via their angular declination. The current studies suggest that egocentric distance perception might be similarly robust to reduced attention when localization is based primarily on a target's angular declination.
\end{abstract}

Keywords Visual selective attention $\cdot$ Spatial attention $\cdot$ Spatial localization

In naturalistic settings, one needs to react to objects that have been glimpsed only briefly or that are not readily distinguished from surrounding clutter. Attention is frequently focused on demanding tasks (e.g., driving, using a smartphone, or even walking on a crowded sidewalk), and thus we are often unable to devote focal attention to objects that must be localized. The consequences of mislocalization can be severe - misreaching for a stable support when one suddenly feels unsteady can result in a serious fall, for example. The aim of this study was to investigate the role of attentional constraints on directional localization, particularly in cases in which objects have been detected but are not within the focus of attention. Under such circumstances, are objects localized less accurately and/or less precisely?

Our focus on directional localization stems from a broader theoretical interest in egocentric distance perception. An object's visual direction when it is resting on the ground is a

John W. Philbeck

philbeck@gwu.edu

1 Department of Psychology, The George Washington University, 2125 G Street, NW, Washington, DC 20052, USA strong cue to its perceived distance (e.g., Gajewski, Philbeck, Wirtz, \& Chichka, 2014; Ooi, Wu, \& He, 2006; Philbeck \& Loomis, 1997). Nearly all studies in the domain of egocentric distance perception have used paradigms that afford focal attention directed to the target's location: There is a single, highly salient and easily detectable target that is seen for several seconds. The impact of reduced attention on directional localization remains poorly understood. Although there is evidence that targets can be localized reasonably well even with minimal attention (Tsal \& Bareket, 1999, 2005), several studies suggest that focal attention can improve both the precision (Tsal \& Bareket, 1999, 2005; Tsal, Meiran, \& Lamy, 1995) and accuracy (Adam, Davelaar, van der Gouw, \& Willems, 2008; Fortenbaugh \& Robertson, 2011) of 2-D localization. Importantly, many of these studies have used brief viewing durations to control the level of attention and preclude eye movements. However, limiting the viewing duration increases the risk that participants might fail to detect the target; thus, when detection is least reliable, at least some of the localization data could be a reflection of guessing, rather than localization per se. To be fair, attentional effects have been observed with longer $(150 \mathrm{~ms})$ viewing durations that presumably support reliable detection (Fortenbaugh \& 
Robertson, 2011). Also, Prinzmetal, Amiri, Allen, and Edwards (1998) only included participants with high detection sensitivity (though this was not evaluated on a trial-by-trial basis) and found that attention improves response precision with 67-ms glimpse durations. Nevertheless, because manipulations of attention would also be expected to affect detection rates, without a trial-by-trial verification of detection, it is unclear to what degree attention's role in localization is mediated by increased frequency of detection.

Work in other domains shows that attention enhances perception at attended locations (Downing \& Pinker, 1985; Eriksen \& Eriksen, 1974; Posner, Snyder, \& Davidson, 1980; Yantis \& Johnston, 1990), and that there are changes in cortical visual representations and neuronal coherence that favor attended locations (e.g., Ben Hamed, Duhamel, Bremmer, \& Graf, 2002; Womelsdorf \& Fries, 2006). Accordingly, we hypothesized that directional localization would be enhanced at the locus of attention, even when errors due to detection failures were minimized. In this view, when targets are presented at viewing durations that support reliable detection, localization performance should be more accurate, and perhaps also more precise, when the viewing conditions supports a higher level of attention. The competing (null) hypothesis is that localization will not differ dramatically depending on whether or not the target is fully attended, as long as the target has been detected. If this outcome holds true, it would be an interesting counterexample to past evidence suggesting that attention enhances perception in general, independent of the task (Carrasco, 2011; Egeth \& Yantis, 1997). On the other hand, the null hypothesis itself has a basis for expectation in the literature. Early research suggested that directional localization is indeed very efficient. Sagi and Julesz (1985), in particular, described a study wherein the time course for the localization of sets of targets followed that for their detection, suggesting to the authors that localization could be done in parallel without focused attention.

There are two additional contributions that our experiment offers. First, we use methods that minimize contamination of localization responses by guessing (i.e., due to detection failures), across a range of brief viewing durations. This provides a particularly precise assessment of attentional effects in 2-D localization. Second, because we focus on directional localization in a vertical plane, the results will have practical relevance for whether or not attention would be expected to influence localization of ground-level targets in the early stages of scene viewing.

Our approach was to precue attention, using exogenous cues, toward or away from the region where a briefly presented target was to appear (Posner, 1980). Observers reported whether the target was detected, and then indicated its location. This allowed for localization performance to be assessed specifically when the target was detected. Also, there were catch trials in which no target was presented, permitting a determination of detection sensitivity across a range of viewing durations (34-150 ms; Experiment 1). These brief durations effectively prevent eye movements and the dynamic shifts of attention that are known to occur during longer stimulus presentations (Astrand, Wardak, Baraduc, \& Ben Hamed, 2016; Dugué, Roberts, \& Carrasco, 2016; Fiebelkorn, Saalmann, \& Kastner, 2013; Laudau \& Fries, 2012), which might otherwise reduce experimental control of the distance between the location of the spatial locus of attention and the target location. Additionally, short cue-to-target time periods ensure that maximal effects of exogenous cuing are observed (Posner, 1980). The fine-grained range of durations in Experiment 1 allowed us to capture temporal changes in detectability and examine localization performance for those durations at which detection had plateaued. Our goals in Experiment 2 were threefold: (1) to isolate performance at $34 \mathrm{~ms}$ and $150 \mathrm{~ms}$, because differences in attention and detection, as evidenced by Experiment 1, are greatest with the former and minimized if not eliminated in the latter; (2) to increase the number of observations for each condition; and (3) to provide internal replication of the finding.

\section{Experiment 1}

\section{Method}

Participants Nineteen undergraduates participated in exchange for course credit. Six of these failed to meet the inclusion criteria (see Results section), yielding a final sample size of 13. This sample size approximately matches that of other studies in this domain, which often range between eight to 16 participants per group (e.g., Adam et al., 2008; Fortenbaugh \& Robertson, 2011; Tsal \& Bareket, 1999, 2005). All observers reported normal or corrected-to-normal vision and provided written consent prior to testing. The study was approved by The George Washington University ethics committee and was performed in accordance with the ethical standards of the 1964 Declaration of Helsinki. All subjects were naïve to the purposes of the study.

Apparatus and design The experiment employed a 2 visual field (upper/lower) $\times 6$ duration $(34-150 \mathrm{~ms}) \times 2$ cue validity (valid/invalid) $\times 15$ eccentricity $\left(2.5^{\circ}-9.5^{\circ}\right)$ factorial design, yielding 360 target-present trials. There were also 10 targetabsent "catch" trials for each of the 12 combinations of target duration and precue location.

The study was conducted on a 24-inch LCD monitor with a refresh rate of $60 \mathrm{~Hz}$. The viewing distance was approximately $60 \mathrm{~cm}$. Testing was in a darkened room, and the border of the monitor was covered with black tape so the display perimeter could not be seen. The target was a single asterisk subtending $0.45^{\circ}$. The target could appear at 30 locations 
along the screen's vertical midline (15 locations above fixation and 15 below), ranging from $2.5^{\circ}$ to $9.5^{\circ}$ from fixation in $0.5^{\circ}$ increments. The precues were two square boxes subtending $9.5^{\circ} \times 9.5^{\circ}$ (see Fig. 1). The centers of the boxes were $5.375^{\circ}$ above and below fixation, and each box fully enclosed all possible target locations on that side of the fixation cross. A dynamic mask was used to disrupt visual processing of the target. The mask consisted of five frames, with each frame presented for $34 \mathrm{~ms}$. Each frame was randomly filled with salt and pepper noise. The mask covered the whole vertical midline of the screen with a width of $0.5^{\circ}$.

Procedure At the beginning of each trial, the two precue boxes and a centered fixation cross were presented for 2,000 ms (see Fig. 1). Observers were asked to maintain fixation at the cross. One box turned red for $50 \mathrm{~ms}$ to provide a valid or invalid cue depending on the location of the upcoming target. Next, the two boxes and the cross disappeared, and a target was presented at one of 30 possible target locations. The target's exposure duration was varied randomly across trials $(34 \mathrm{~ms}, 50 \mathrm{~ms}, 67$ $\mathrm{ms}, 84 \mathrm{~ms}, 100 \mathrm{~ms}$, and $150 \mathrm{~ms})$. Then, the dynamic mask was

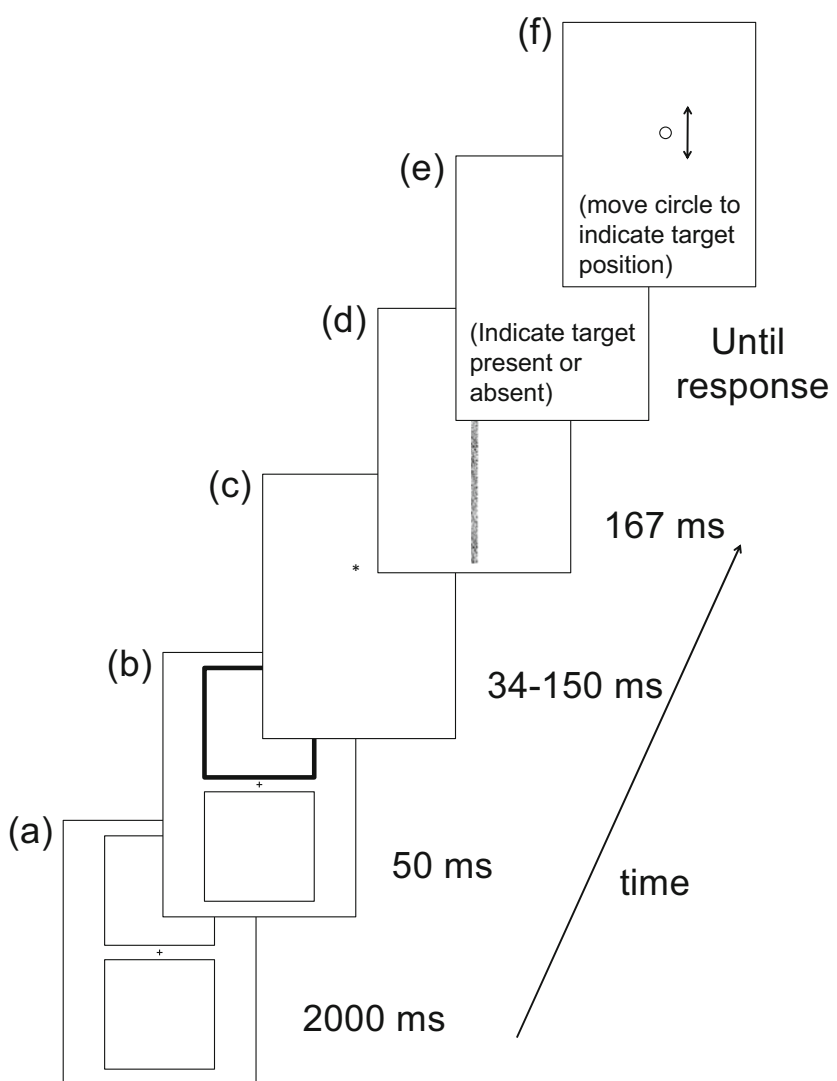

Fig. 1 Sequence of events in a typical trial. Outer rectangles in each panel were not visible to the observer. a Trial begins with pre-cue boxes and a fixation cross. b One box turns red (shown here as a thicker line) to cue attention. c Target is presented at one of the 30 possible target locations. $d$ A dynamic mask appears. e Observers indicate target present or absent. f Observers use mouse to indicate target position. Text in panels e and $\mathbf{f}$ was not visible to observers presented for $167 \mathrm{~ms}$. After the mask disappeared, observers judged whether the target was present or absent (left or right mouse click, respectively). Observers were instructed to indicate that the target was present "only if they were absolutely positive." Regardless of response, a circle was then presented at the center of the screen. Observers used the mouse to move the circle to where they thought the target was presented and then clicked the left button. If observers indicated that there was a target when in fact no target was presented, a "no target" feedback message appeared in the middle of the screen after they had positioned the circle. Otherwise, no feedback was given.

\section{Results and discussion}

Participants with a false-alarm rate more than two standard deviations above the mean in detecting the targets were removed prior to further analysis to minimize contamination of the localization estimates by trials in which targets were not detected. Additionally, participants with a hit rate more than two standard deviations below the mean and those who failed to get at least three hits in every condition (across all eccentricities) were removed from analysis. Exceptionally high miss rates diminish the ability to estimate parameters (slopes, mean signed errors, and standard errors of estimate). In total, six out of 19 observers failed to meet these criteria and were removed from the analysis. All subsequent analyses were conducted with incorrect ("miss") responses removed. Across the 13 observers included in the final analysis, the percentage of trials removed due to misses ranged from $1 \%$ to $25 \%$ (mean $=$ $8.7 \%$ ). Somewhat more trials were removed at the briefest viewing duration (mean $=2.9 \%$ ) than at the longer durations (means $=1.6 \%, 1.1 \%, 1.1 \%, 1.0 \%$ and $1.0 \%$ for $50 \mathrm{~ms}, 67 \mathrm{~ms}$, $84 \mathrm{~ms}, 100 \mathrm{~ms}$, and $150 \mathrm{~ms}$, respectively). There was only a very weak positive correlation between number of trials removed and eccentricity $(r=.07, p=.83$ ), suggesting that misses were approximately equally distributed across eccentricities.

Detection The average false alarm rate was $4.6 \%$; false alarms were equally distributed between upper and lower visual field (averaging 2.77 false alarms in each hemifield out of 120 total catch trials per participant). Detection performance was analyzed based on the sensitivity index $\left(d^{\prime}: z\right.$ (hits) $-z$ (false alarms)) for each condition. For conditions with hit rates of 1.0 , values of 1 were replaced with $(N-.5) / N$, where $N$ is the total number of each participant's hit/false-alarm trials for that condition; for conditions with false-alarm rates of zero, values of zero were replaced with $0.5 / N$ (Macmillan \& Kaplan, 1985). Note that it was not possible to assign catch trials to some conditions - for example, valid and invalid cue conditions were defined with reference to the target location, but for 
catch trials, cue validity is undefined as there was no target. For such conditions, we used $0.5 / \mathrm{N}$ as the false-alarm rate when calculating $d$-prime. Data were collapsed across eccentricity to get $d$-prime estimates for each participant. This analysis determined the time course of the cuing effect on target detection (see Fig. 2a). These data were subjected to repeatedmeasures analysis of variance (ANOVA; see Table 1). There was a main effect of viewing duration, with higher $d$-prime at longer viewing durations, and a main effect of cue validity, with higher $d$-prime for validly cued targets; these significantly interacted. Two-tailed, paired-samples $t$ tests comparing valid and invalid cue conditions showed that the Cue Validity $\times$ Viewing Duration interaction was driven by significant cue validity effects at $34 \mathrm{~ms}, t(12)=-5.15, p=.001, d=$ -1.43 , and $84 \mathrm{~ms}, t(12)=-3.57, p=.004, d=-0.99$. Additionally, the ANOVA revealed a main effect of visual field, such that detection sensitivity was higher in the upper than in the lower visual field $\left(d^{\prime}=2.97\right.$ versus 2.72 , respectively).

Given the stated aims of examining the influence of attention on localization during brief versus longer glimpses, and to provide a basis for comparison to Experiment 2, we ran another ANOVA comparing detection performance for the two extreme exposure durations ( $34 \mathrm{~ms}$ and $150 \mathrm{~ms}$ ). This yielded the same pattern as did the analysis that included all exposure durations, with main effects of visual field, cue validity, and viewing duration. It also yielded the same significant Viewing Duration $\times$ Cue Validity interaction. The pattern of results confirms a maximal attention effect at the 34-ms duration and stable performance with high detection rates at the 150 $\mathrm{ms}$ duration. The significant cue validity effects additionally confirm the efficacy of our exogenous cuing manipulation.

Our subsequent analyses focused on comparing localization performance at the two extreme viewing durations ( $34 \mathrm{~ms}$ versus $150 \mathrm{~ms})$. Localization performance was characterized by (1) response sensitivity - the slope of the observer's localization responses in each condition as a function of the actual target eccentricity; (2) bias - the overall tendency to underestimate or overestimate target eccentricity, reported here via mean signed errors (MSEs); and (3) response precisionestimated by the standard errors of estimates (SEEs), which is the spread of data around each observer's best fitting regression line for each viewing condition. For each measure, we only included trials in which participants reported seeing the target. Slopes, MSEs, and SEEs were subjected to repeatedmeasures ANOVAs.

Response sensitivity Fig. 3 shows location judgments as a function of target eccentricity. Response sensitivity was generally high, with slopes near 1 in all conditions. The ANOVA on slopes showed no main effects of either viewing duration or cue validity and no reliable interactions (see Table 1). In particular, response sensitivity was marginally greater in the 150 -ms viewing condition (mean slope $=0.95$ ) than in the 34$\mathrm{ms}$ viewing condition (mean slope $=0.85$ ), $p=.058$. Similarly, response sensitivity was marginally greater in the invalid cue condition (mean slope $=0.93$ ) than in the valid condition (mean slope $=0.87) p=.086$. Finally, the Cue Validity $\times$ Visual Field interaction also approached, but did not reach, the level of statistical significance, $p=.073$. In the upper visual field, the mean slopes for valid and invalid cue conditions were 0.86 and 0.85 , respectively, whereas for the lower visual field, the mean slopes were somewhat higher in the invalid (1.01) than in the valid (0.87) conditions. There were no other effects or interactions (all other $F \mathrm{~s}<2.34$ and all other $p \mathrm{~s}>.15$ ).

Bias Peripheral biases were coded as positive and foveal biases as negative. Table 1 shows that there was a main effect of cue validity, indicating greater bias in the valid than the invalid cue condition (MSE $=1.00 \mathrm{~cm}$ vs $0.73 \mathrm{~cm}$, respectively). There was also a main effect of visual field, indicating greater bias in the lower than the upper visual field (MSE $=1.34 \mathrm{~cm}$ vs. $0.40 \mathrm{~cm}$, respectively). This effect was qualified by a significant Visual Field $\times$ Viewing Duration interaction: two-tailed $t$ tests showed

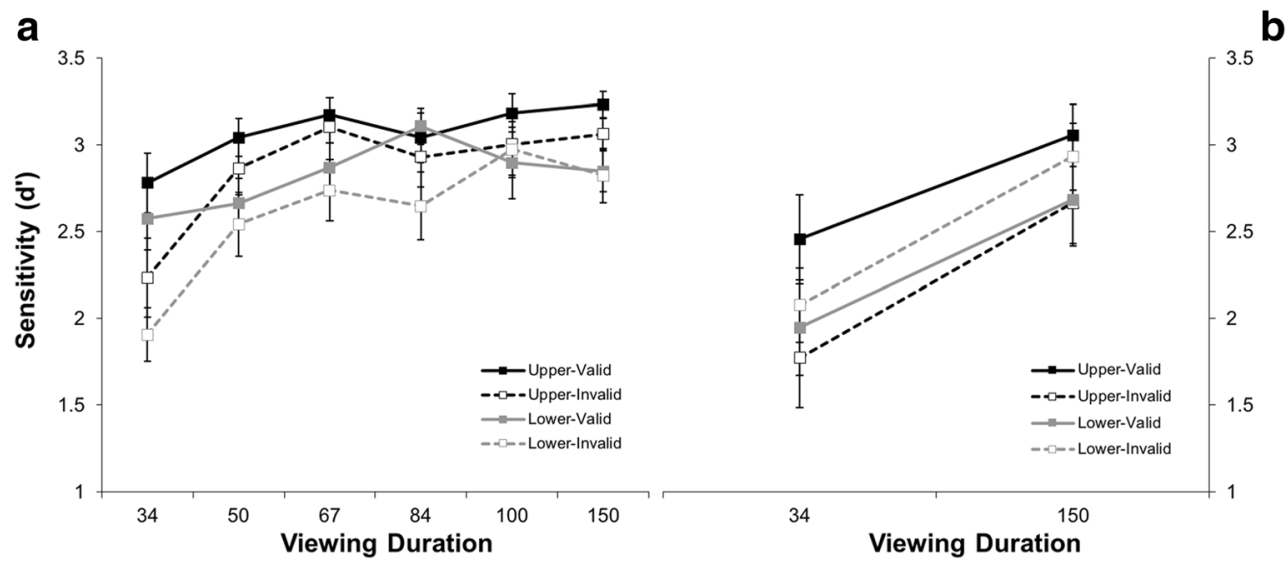

Fig. 2 Mean $d$-prime values as a function of viewing duration (in milliseconds), visual field (upper versus lower) and cue validity (valid versus invalid) for Experiment 1 (a) and Experiment 2 (b). Error bars show \pm 1 standard error of the mean 
Table 1 Analysis of variance results for Experiments 1 and 2

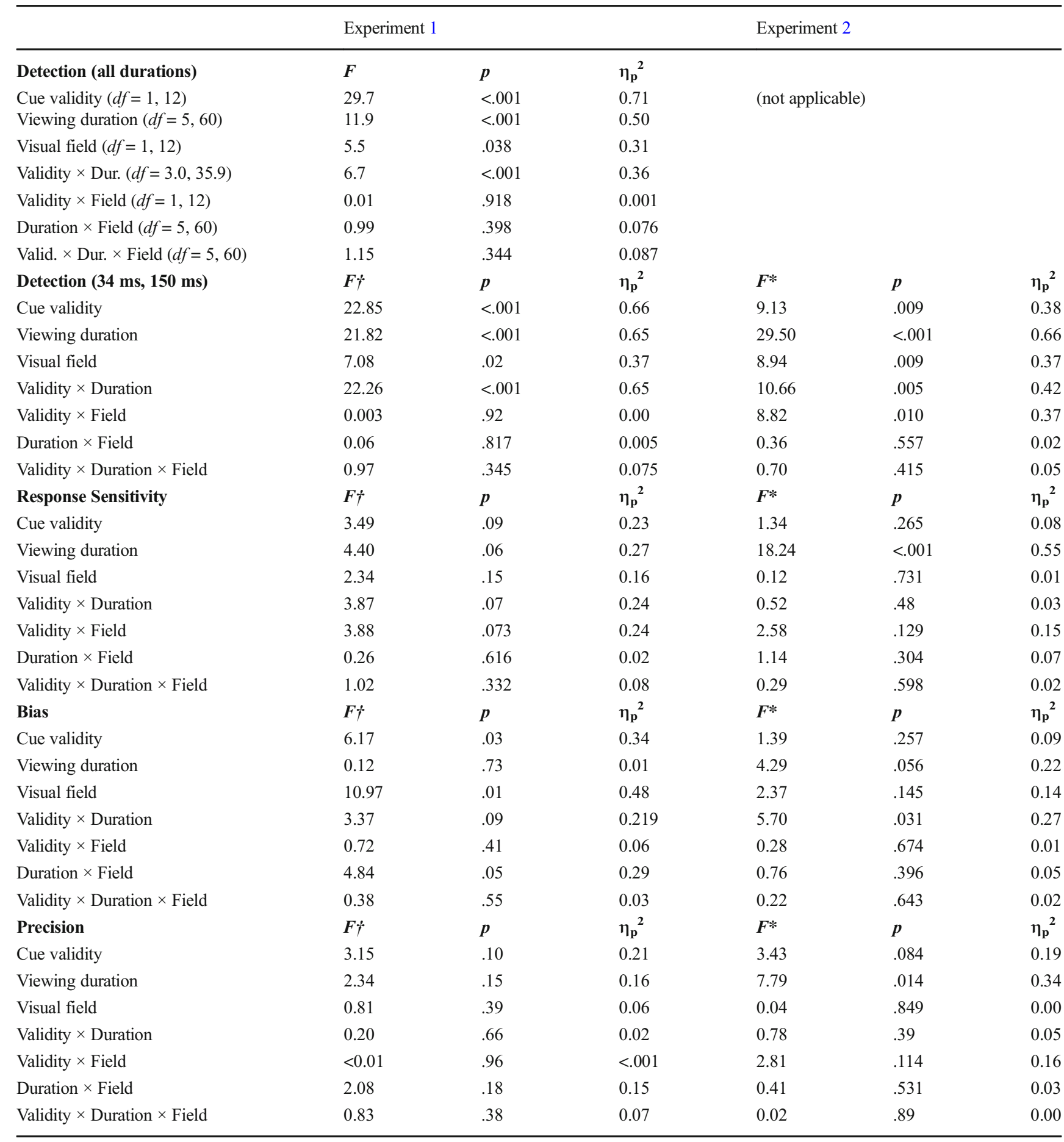

$\dagger d f=1,12$

$* d f=1,15$

that overestimation bias reliably increased in the upper visual field going from $34 \mathrm{~ms}$ to $150 \mathrm{~ms}$ viewing durations (MSE $=0.26 \mathrm{~cm}$ versus $0.53 \mathrm{~cm}$, respectively), $t(12)=-3.77, p=.003, d=-1.05$, while bias reliably decreased in the lower visual field going from $34 \mathrm{~ms}$ to $150 \mathrm{~ms}$ viewing durations (MSE $=1.42 \mathrm{~ms}$ versus 1.26 $\mathrm{cm}$, respectively); $t(12)=-2.47, p=.03, d=-0.68$. There were no other effects or interactions. The most nearly reliable of these was a marginal Viewing Duration $\times$ Cue Validity interaction $(F=$ 3.37 and $p=.09$ ), which reflected a slightly larger cue validity effect for the $34 \mathrm{~ms} /$ upper visual field condition. 


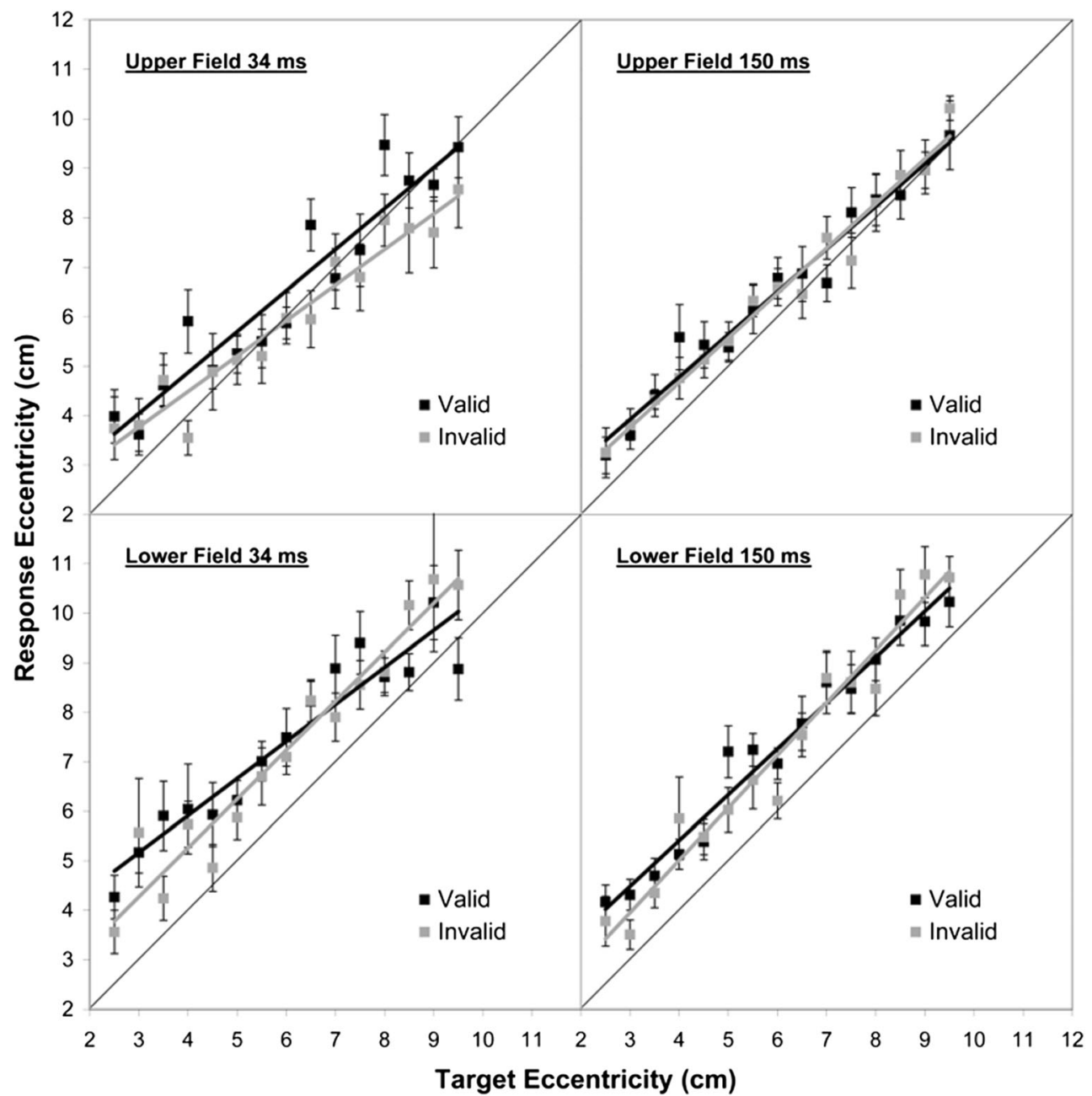

Fig. 3 Mean indicated target location in Experiment 1 as a function of visual field (upper versus lower), cue validity (valid versus invalid), target duration (34 versus $150 \mathrm{~ms}$ ), and target eccentricity. For Lower Field

Precision Responses were slightly more precise (smaller SEEs) in the valid than in the invalid cue condition $(1.12 \mathrm{~cm}$ and $1.27 \mathrm{~cm}$, respectively), and more precise with longer than shorter glimpses $(1.14 \mathrm{~cm}$ and $1.25 \mathrm{~cm}$, respectively). However, neither main effect was statistically reliable $\left(F_{\mathrm{S}}<\right.$ 3.154 and $p s>.10$; see Table 1$)$. There were no other effects or interactions $(F \mathrm{~s}<2.079$ and $p \mathrm{~s}>.17)$.

Perhaps the most striking outcome of Experiment 1 was the upper field advantage that appeared in the detection analyses. While there is evidence that the resolution of attention is fine grained in the lower visual field (He, Cavanagh, \& Intriligator, 1996), there are also extant data showing performance advantages in the upper visual field (Levine \& McAnany, 2005). Previc (1990) has suggested that while resolving power is enhanced in the lower field because fine discriminations are more prevalent for items within hand's reach, shifts of attention to the upper field are more rapid to support orientation to distal stimuli. Here, localization performance did not suggest a lower visual field benefit in terms of discrimination (i.e., panels, increasing target eccentricity denotes increasing declination below fixation point. At the viewing distance used in this study, $1 \mathrm{~cm} \approx$ $1^{\circ}$. Error bars show \pm 1 standard error of the mean

response sensitivity), but there was a tendency for targets in the lower visual field to be localized farther away from the fixation point than targets in the upper visual field. A similar tendency has recently been found (Fortenbaugh, Robertson, \& Esterman, 2017) using a methodology that differs along a number of potentially relevant dimensions (e.g., lack of visible borders, larger eccentricities, endogenous rather than exogenous attentional cueing, longer viewing durations relative to our study). Also, our finding of greater bias in valid than in invalid trials mirrors results of Yeshurun and Carrasco (1998), who found that focal attention impaired performance for relative central locations in a texture segregation task involving a field of 287 elements. Although the similarity in results may reflect generalizable effects across experimental contexts, the many methodological differences present challenges for direct comparisons. Overall, however, localization performance did not suggest much, if any, cost for localization in the conditions where the level of attention was diminished. 


\section{Experiment 2}

One feature of Experiment 1 is that, in the interest of testing across multiple viewing durations while keeping the number of trials manageably low, we obtained only one measurement per condition. This could yield increased response variance relative to other designs that have collected multiple measurements per condition (e.g., Prinzmetal et al., 1998, 2005). Also, as noted above, somewhat more trials were omitted in Experiment 1 due to missed targets at the briefest duration than at the longer durations. To obtain a more robust assessment of our research questions, we tested another group of participants using a very similar design and procedure as in Experiment 1, except that here we compared only the $34 \mathrm{~ms}$ and $150 \mathrm{~ms}$ viewing durations and collected five measurements per condition. This design has two advantages in that it allows us to (1) increase the reliability of measurement, by increasing the number of replications for the critical intervals of $34 \mathrm{~ms}$ and $150 \mathrm{~ms}$ exposure durations; and (2) provide internal replication of our findings.

\section{Method}

Participants Nineteen undergraduates participated in exchange for $\$ 8$ cash. Three of these failed to meet the inclusion criteria (same criteria as in Experiment 1), yielding a final sample size of 16 .

Apparatus, design, and procedure The apparatus, design, and procedure were the same as in Experiment 1, with the exception that here, we only used viewing durations of $34 \mathrm{~ms}$ and $150 \mathrm{~ms}$, and we measured each condition five times apiece instead of once apiece. There were 10 target-absent "catch" trials for each of the four combinations of duration and precue location per repetition per condition. This design yielded 600 experimental trials and 200 catch trials per participant. Across the 16 observers included in the final analysis, the percentage of trials removed due to misses ranged from $0.5 \%$ to $47.9 \%$ (mean $=15.9 \%)$. There was a weak but unreliable negative correlation between number of trials removed and eccentricity $(r=-.44, p=.10)$, suggesting that misses were approximately equally distributed across eccentricities.

\section{Results and discussion}

Detection The average false alarm rate was $10.6 \%$; here, there were more false alarms in the lower visual field $(M=13.7)$ than in the upper $(M=7.4) ; t(15)=2.16, p=.047, d=.541$, out of 200 total catch trials per participant. Otherwise, the results largely mirrored those of Experiment 1 (see Fig. 2 and Table 1). There was a main effect of viewing duration, with higher $d$-prime at longer viewing durations, and a main effect of cue validity, with higher $d$-prime for validly cued targets; these significantly interacted. Two-tailed $t$ tests showed that the Cue Validity $\times$ Viewing Duration interaction was driven by significant cue validity effects at $34 \mathrm{~ms}$ durations, $t(15)=3.95, p=.001, d=.988$, while there was no difference between validity conditions at $150 \mathrm{~ms}, t(15)=$ $1.17, p=.260, d=.293$. The significant cue validity effects again confirm the efficacy of our exogenous cue. Additionally, the ANOVA revealed a main effect of visual field, with higher detection sensitivity in the upper $\left(d^{\prime}=2.48\right)$ than the lower $\left(d^{\prime}\right.$ $=2.41$ ) visual field. Unlike in Experiment 1, here there was a significant Visual Field $\times$ Cue Validity interaction. A twotailed $t$ test revealed that this was driven by significant cue validity effects in the upper visual field, $t(15)=4.04, p=.001$, $d=1.010$, with no validity-based difference in the lower visual field, $t(15)=1.38, p=.189, d=.344$.

Response sensitivity Figure 4 shows the mean data of Experiment 2 across all conditions. The ANOVA on slopes showed a main effect of viewing duration, with a higher mean slope for the $150 \mathrm{~ms}(M=.89)$ than for the $34 \mathrm{~ms}$ viewing duration $(M=.66)$. This was a change relative to Experiment 1 , in which the effect of duration was marginally significant, $p$ $=.06$. No other effects or interactions reached significance (see Table 1).

Bias There was a marginal main effect of viewing duration $(p$ $=.056$ ), with less bias in the shorter duration (an average bias of $-0.13 \mathrm{~cm}$ - toward the fovea) than the longer duration (an average bias of $0.34 \mathrm{~cm}$ - toward the periphery). There was no such effect in Experiment 1. There was a significant Validity $\times$ Viewing Duration interaction, such that bias generally increased with duration, but increased more for invalidly cued than validly cued targets, averaging $+0.70 \mathrm{~cm}$ versus +0.25 $\mathrm{cm}$, respectively. (This interaction was not significant in Experiment 1, at $p=.09$.) There were no other significant main effects or interactions (all other $F \mathrm{~s}<2.37$, and all other $p$ s $>$.145). Interestingly, several contrasts that were significant in Experiment 1 did not reach significance in Experiment 2 (see Table 1); specifically, the main effects of cue validity and visual field in Experiment 1 disappeared in Experiment 2, as did the marginal $(p=.05)$ Duration $\times$ Visual Field interaction in Experiment 1.

Precision Unlike in Experiment 1, here there was a main effect of viewing duration, with more precise responses (smaller SEEs) in longer durations than shorter durations $(0.69 \mathrm{~cm}$ and $0.98 \mathrm{~cm}$, respectively). There was a marginally significant main effect of validity, $p=.084$, with more precise responses in the valid than in the invalid condition $(0.79 \mathrm{~cm}$ and $0.87 \mathrm{~cm}$, respectively). No other effects or interactions reached significance $(F \mathrm{~s}<2.81$ and $p \mathrm{~s}>.114)$.

As we have shown, the results differed somewhat between Experiments 1 and 2, despite the methodologies being 


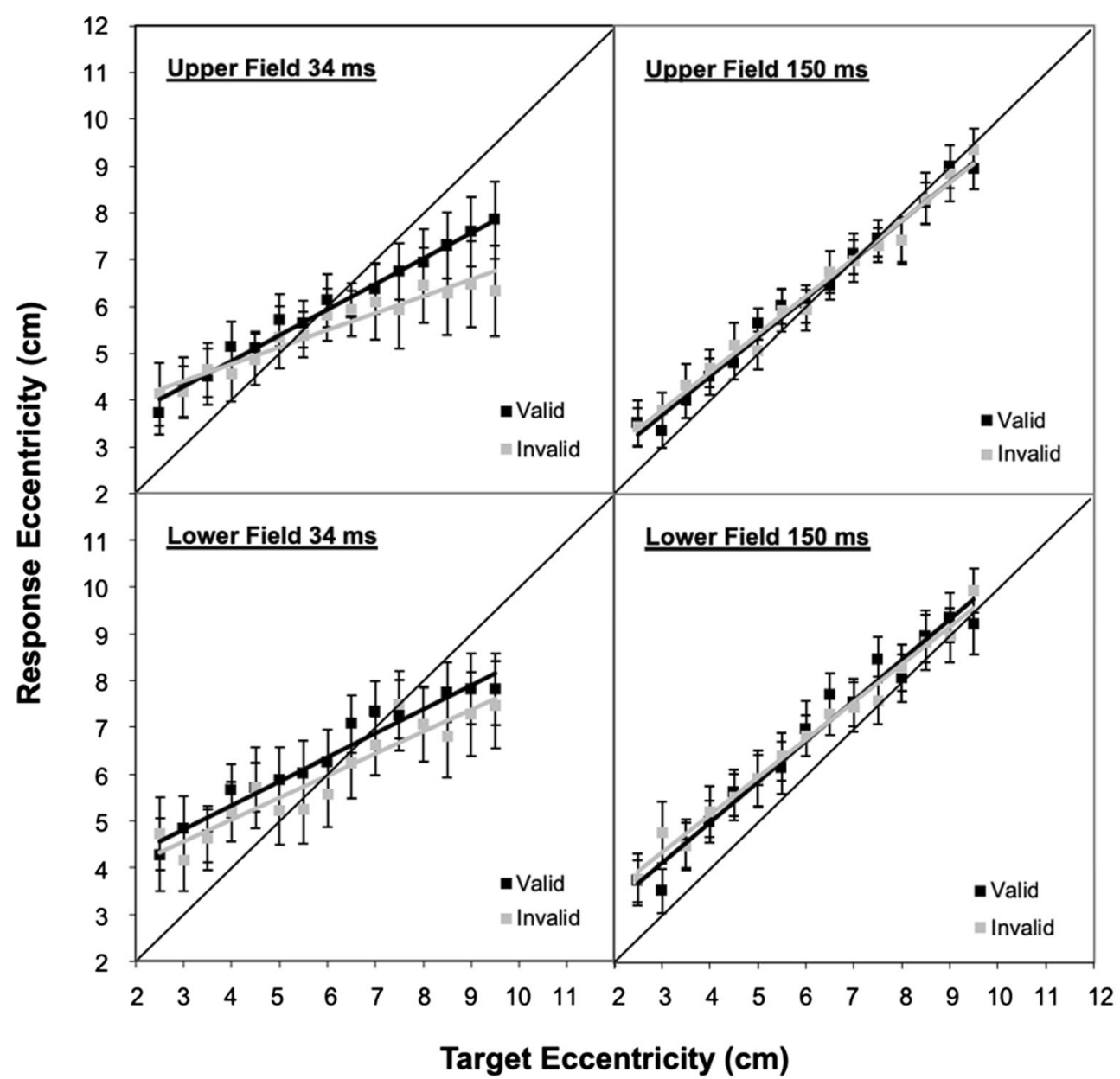

Fig. 4 Mean indicated target location as a function of visual field (upper versus lower), cue validity (valid versus invalid), target duration (34 versus $150 \mathrm{~ms}$ ), and target eccentricity for Experiment 2. For Lower
Field panels, increasing target eccentricity denotes increasing declination below fixation point. At the viewing distance used in this study, $1 \mathrm{~cm} \approx 1^{\circ}$. Error bars show \pm 1 standard error of the mean

\section{General discussion}

nificant effect of viewing duration on response sensitivity and marginal effect $(p=.56)$ on bias. Because viewing duration had the biggest impact on detection rates, it is likely that targets benefitted from an enhanced level of attention in the $150 \mathrm{~ms}$ versus $34 \mathrm{~ms}$ conditions. Presumably, the effect of duration indicates a nonspatially specific effect of attention. However, the significant interaction between viewing duration and cue validity in the analysis of bias indicates some additional effect of spatial attention elicited by the validity manipulation. The results of Experiment 2 therefore suggest at least some impairment in localization outside the focus of attention, even when included responses were all confidently detected.

The primary change was that Experiment 2 used only the briefest and longest durations and collected five measurements per condition. This afforded more robust estimates of response sensitivity, bias and precision and likely explains any differences in results between the two studies. It also highlights the importance of obtaining multiple samples in this paradigm.
Our hypothesis was that directional localization is enhanced at the spatial focus of attention when targets have been detected. This view predicts that there should be cue validity effects in terms of the accuracy and/or precision of responses, even when detection sensitivity is high. We addressed this prediction by varying cue validity and viewing duration, measuring detection sensitivity across conditions, and restricting analysis to target-present trials in which participants indicated they had seen the target. The answer to whether or not this prediction was supported in our paradigm depended on the amount of data collected per condition. In Experiment 1, with one measurement per condition, responses were generally biased toward the periphery, even under conditions that maximized target detectability (i.e., validly-cued targets seen at the longest viewing durations). Experiment 1 also saw greater bias in validly cued conditions (mirroring Yeshurun \& Carrasco, 1998, using somewhat different methods). Experiment 2, meanwhile, involved five measurements per condition and a 
more restricted set of viewing durations, and although validly cued targets were detected more readily than invalidly cued targets in that experiment, they generally did not benefit relative to invalidly cued targets in terms of response sensitivity, bias, or precision. There was, however, a significant Validity $\times$ Duration interaction in the bias analysis of Experiment 2, indicating that localization does benefit somewhat at the spatial focus of attention when viewing durations are very brief; in this case, invalidly cued targets were localized with some bias in the foveal direction, which happened to be in the direction of the cue in Experiment 2.

Several reports suggest that spatial representations can be distorted by attention and/or the presence of contours and display elements (e.g., Liverence \& Scholl, 2011; Ono \& Watanabe, 2011; Suzuki \& Cavanagh, 1997; Yamada, Kawabe, \& Miura, 2008), such that areas around the fovea and near the locus of attention tend to be overrepresented relative to surrounding space (Kravitz \& Behrmann, 2008; Wardak et al., 2011). In addition, some past studies have shown a similar robustness of localization precision to attentional manipulations (e.g., Suzuki \& Cavanagh, 1997; Yamada et al., 2008), while others have shown an increase in precision associated with focal attention (e.g., Adam et al., 2008; Adam, Ketelaars, Kingma, \& Hoek, 1993; Tsal $\&$ Bareket, 1999, 2005). These studies differ in many details, depending upon their specific research questions, and as we outlined earlier, they have generally not assessed detectability on a trial-by-trial basis. Nevertheless, our primary objective was to determine whether directional localization performance for reliably detected targets would benefit from focal attention to the target region compared with performance when the target appeared outside the focus of attention. Our data suggest that, although very brief viewing durations do negatively affect localization via generalized attentional effects, localization generally does not benefit at the spatial focus of attention, at least at the viewing durations and eccentricities used in the current study. Localization was somewhat less biased at very brief durations when targets were validly cued than invalidly cued (averaging 0.06 vs. $-0.31 \mathrm{~cm}$, respectively); though statistically robust, these differences were quite small. In sum, while directional localization can be at least modesty improved with attention, it is nevertheless quite efficient, as would be expected by works pointing to location as an obligatory component in visual representation (e.g., Huang \& Pashler, 2007; Huang, Treisman, \& Pashler, 2007; Johnston \& Pashler, 1990). An important implication of this study for 3 $\mathrm{D}$ visual space perception is that the kind of 2-D directional information required to locate distal targets along the ground (in 3-D) can indeed be extracted from brief glimpses even with reduced attention. All levels of performance were associated with some response sensitivity (slopes significantly greater than zero) and although Experiment 1 did exhibit some peripheral biases, responses in Experiment 2 were not largely biased (MSEs were neither significantly greater than nor significantly less than zero), $p s<.05$. Furthermore, there was at most only a marginal validity-related difference $(p=.08)$ in the standard errors of estimate, which suggests relatively little benefit of spatial attention in terms of precision, provided that the target has been detected. Taken together, our results highlight the remarkable robustness of the human ability to extract information about visual direction. This has implications for real-world visual control of locomotion. As we walk, we fixate (and pay attention to) a succession of objects in the environment, but we do this selectively. We cannot fixate all objects that might be behaviorally relevant in our upcoming path. Although this idea remains to be confirmed in future work, one implication of the current results is that 3-D localization of objects on the nearby ground plane is likely not affected in a substantial way when attention is directed elsewhere, provided that the objects have been detected. This robustness would have clear functional utility.

Author note Daniel A. Gajewski, Junjun Zhang, Sarah Shomstein, Joseph C. Nah and John W. Philbeck, Department of Psychology, The George Washington University, Washington, DC.

Research reported in this publication was supported by the National Eye Institute of the National Institutes of Health under Award Number R01EY021771. The content is solely the responsibility of the authors and does not necessarily represent the official views of the National Institutes of Health.

\section{References}

Adam, J. J., Davelaar, E. J., van der Gouw, A., \& Willems, P. (2008). Evidence for attentional processing in spatial localization. Psychological Research, 72, 433-442.

Adam, J. J., Ketelaars, M., Kingma, H., \& Hoek, T. (1993). On the time course and accuracy of spatial localization: Basic data and a twoprocess model. Acta Psychologica, 84, 135-159.

Astrand, E., Wardak, C., Baraduc, P., \& Ben Hamed, S. (2016). Direct two-dimensional access to the spatial location of covert attention in macaque prefrontal cortex. Current Biology, 26, 1699-1704.

Ben Hamed, S., Duhamel, J.-R., Bremmer, F., \& Graf, W. (2002). Visual receptive field modulation in the lateral intraparietal area during attentive fixation and free gaze. Cerebral Cortex, 12, 234-245.

Carrasco, M. (2011). Visual attention: The past 25 years. Vision Research, $51,1484-1525$.

Downing, C., \& Pinker, S. (1985). The spatial structure of visual attention. In M. I. Posner \& O. S. M. Marin (Eds.), Attention and performance XI (pp. 171-188). Hillsdale, NJ: Erlbaum.

Dugué, L., Roberts, M., \& Carrasco, M. (2016). Attention reorients periodically. Current Biology, 26, 1595-1601.

Egeth, H. E., \& Yantis, S. (1997). Visual attention: Control, representation, and time course. Annual Review of Psychology, 48, 269-297.

Eriksen, B. A., \& Eriksen, C. W. (1974). Effects of noise letters upon the identification of a target letter in a nonsearch task. Perception \& Psychophysics, 16, 143-149.

Fiebelkorn, I. C., Saalmann, Y. B., \& Kastner, S. (2013). Rhythmic sampling within and between objects despite sustained attention at a cued location. Current Biology, 23, 2553-2558. 
Fortenbaugh, F. C., Robertson, L. C. (2011). When here becomes there: Attentional distribution modulates foveal bias in peripheral localization. Attention, Perception, \& Psychophysics, 73, 809-828.

Fortenbaugh, F. C., Robertson, L. C., \& Esterman, M. (2017). Changes in the distribution of sustained attention alter the perceived structure of visual space. Vision Research, 131, 26-36.

Gajewski, D. A., Philbeck, J. W., Wirtz, P. W., \& Chichka, D. (2014). Angular declination and the dynamic perception of egocentric distance. Journal of Experimental Psychology: Human Perception \& Performance, 40, 361-377.

He, S., Cavanagh, P., \& Intriligator, J. (1996). Attentional resolution and the locus of visual awareness. Nature, 383, 334-337.

Huang, L., \& Pashler, H. (2007). A Boolean map theory of visual attention. Psychological Review, 114, 599-631.

Huang, L., Treisman, A., \& Pashler, H. (2007). Characterizing the limits of human visual awareness. Science, 317, 823-825.

Johnston, J. C., \& Pashler, H. (1990). Close binding of identity and location in visual feature perception. Journal of Experimental Psychology: Human Perception and Performance, 16, 843-856.

Kravitz, D. J., \& Behrmann, M. (2008). The space of an object: Object attention alters the spatial gradient in the surround. Journal of Experimental Psychology: Human Perception and Performance, 34, 298-309.

Laudau, A. N., \& Fries, P. (2012). Attention samples stimuli rhythmically. Current Biology, 22, 1000-1004.

Levine, M. W., \& McAnany, J. J. (2005). The relative capabilities of the upper and lower visual hemifields. Vision Research, 45, 2820-2830.

Liverence, B. M., \& Scholl, B. J. (2011). Selective attention warps spatial representation: Parallel but opposing effects on attended versus inhibited objects. Psychological Science, 22, 1600-1608.

Macmillan, N. A., \& Kaplan, H. L. (1985). Detection theory analysis of group data: Estimating sensitivity from average hit and false-alarm rates. Psychological Bulletin, 98(1), 185-199.

Ono, F., \& Watanabe, K. (2011). Attention can retrospectively distort visual space. Psychological Science, 22, 472-477.

Ooi, T. L., Wu, B., \& He, Z. J. (2006). Perceptual space in the dark affected by the intrinsic bias of the visual system. Perception, 35 , 605-624.

Philbeck, J. W., \& Loomis, J. M. (1997). Comparison of two indicators of perceived egocentric distance under full-cue and reduced-cue conditions. Journal of Experimental Psychology: Human Perception \& Performance, 23, 72-85.

Posner, M. I. (1980). Orienting of attention. The Quarterly Journal of Experimental Psychology, 32, 3-25.

Posner, M. I., Snyder, C. R., \& Davidson, B. J. (1980). Attention and the detection of signals. Journal of Experimental Psychology: General, 109, 160-174.

Previc, F. H. (1990). Functional specialization in the lower and upper visual fields in humans: Its ecological origins and neurophysiological implications. Behavioral and Brain Sciences, 13, 519-575.
Prinzmetal, W., Amiri, H., Allen, K., \& Edwards, T. (1998). Phenomenology of attention: I. Color, location, orientation, and spatial frequency. Journal of Experimental Psychology: Human Perception \& Performance, 24, 261-282.

Prinzmetal, W., McCool, C., \& Park, S. (2005). Attention: reaction time and accuracy reveal different mechanisms. Journal of Experimental Psychology: General, 134, 73-92.

Sagi, D., \& Julesz, B. (1985). "Where" and "what" in vision. Science, $228,1217-1219$.

Suzuki, S., \& Cavanagh, P. (1997). Focused attention distorts visual space: An attentional repulsion effect. Journal of Experimental Psychology: Human Perception \& Performance, 23, 443-463.

Tsal, Y., \& Bareket, T. (1999). Effects of attention on localization of stimuli in the visual field. Psychonomic Bulletin \& Review, 6 , 292-296.

Tsal, Y., \& Bareket, T. (2005). Localization judgments under various levels of attention. Psychonomic Bulletin \& Review, 12, 559-566.

Tsal, Y., Meiran, N., \& Lamy, D. (1995). Towards a resolution theory of visual attention. Visual Cognition, 2, 313-330.

Wardak, C., Deneve, S., \& Ben Hamed, S. (2011). Focused visual attention distorts distance perception away from the attentional locus. Neuropsychologia, 29, 535-545.

Womelsdorf, T., \& Fries, P. (2006). Neuronal coherence during selective attentional processing and sensory-motor integration. Journal of Physiology-Paris, 100, 182-193.

Yamada, Y., Kawabe, T., \& Miura, K. (2008). Mislocalization of a target toward subjective contours: Attentional modulation of location signals. Psychological Research, 72, 273-280.

Yantis, S., \& Johnston, J. C. (1990). On the locus of visual selection: Evidence from focused attention tasks. Journal of Experimental Psychology: Human Perception \& Performance, 16, 135-149.

Yeshurun, Y., \& Carrasco, M. (1998). Attention improves or impairs visual performance by enhancing spatial resolution. Nature, 396, $72-72$.

The data and materials for all experiments reported here are available through electronic mail addressed to the corresponding author; none of the experiments was preregistered.

Publisher's note Springer Nature remains neutral with regard to jurisdictional claims in published maps and institutional affiliations. 